UT-819

July, 1998

\title{
Fluctuation-dissipation theorem and quantum tunneling with dissipation at finite temperature
}

\author{
Kazuo Fujikawa and Hiroaki Terashima \\ Department of Physics, University of Tokyo \\ Bunkyo-ku, Tokyo 113, Japan
}

\begin{abstract}
A reformulation of the fluctuation-dissipation theorem of Callen and Welton is presented in such a manner that the basic idea of Feynman-Vernon and Caldeira -Leggett of using an infinite number of oscillators to simulate the dissipative medium is realized manifestly without actually introducing oscillators. If one assumes the existence of a well defined dissipative coefficient $R(\omega)$ which little depends on the temperature in the energy region we are interested in, the spontanous and induced emissions as well as induced absorption of these effective oscillators with correct Bose distribution automatically appears. Combined with a dispersion relation, we reproduce the tunneling formula in the presence of dissipation at finite temperature without referring to an explicit model Lagrangian. The fluctuation-dissipation theorem of Callen-Welton is also generalized to the fermionic dissipation (or fluctuation) which allows a transparent physical interpretation in terms of second quantized fermionic oscillators. This fermionic version of fluctuation-dissipation theorem may become relevant in the analyses of, for example, fermion radiation from a black hole and also supersymmetry at the early universe.
\end{abstract}

( Physical Review E, in press) 


\section{Introduction}

The fluctuation-dissipation theorem, which relates the spontaneous fluctuation of "force fileds" in thermal equilibrium to irreversible dissipation, provides a basis of statistical mechanics for irreversible processes which are slightly away from thermal equilibrium. The fluctuation-dissipation theorem has been formulated by various authors in the past [1] - [7]. We find the formulation by Callen and Welton 2] intuitively understandable and appealing. They showed[2] that a general form of fluctuation-dissipation theorem covers a wide range of phenomena such as the Einstein relation for Brownian motion [8], the Nyquist formula for voltage fluctuation in conductors [9], and the Planck distribution for photons. In Ref. 10, it was shown that the effect of dissipation on quantum tunneling ( or coherence ) at zero-temperature can be formulated on the basis of the fluctuation-dissipation theorem of CallenWelton and causality and unitarity (i.e., dispersion relations) without referring to an explicit form of Lagrangian a la Caldeira and Leggett[11].

In this paper, we present a reformulation of the fluctuation-dissipation theorem of Callen and Welton in such a manner that the basic idea of Feynman-Vernon [0] and Caldeira -Leggett [1], which simulates the dissipative medium by an infinite number of oscillators, becomes manifest without actually introducing oscillators. The quantum tunneling at finite temperature is described by this reformulation. Although we use the quantum mechanical Fermi's golden rule, the spontanous and induced emissions as well as induced absorption of these effective oscillators with correct Bose distribution automatically appears, if one assumes the existence of a well defined dissipative coefficient $R(\omega)$ which little depends on the temperature in the energy region we are interested in.

To be specific, for the simplest case of a hermitian (composite) operator

$Q$, which represents the ( generally complicated ) dynamical freedom of the dissipative medium, we have relations

$$
\frac{2}{\pi} \frac{\hbar \omega}{2}\left[1+\frac{1}{e^{\beta \hbar \omega}-1}\right] R(\omega)=\hbar \int_{0}^{\infty} d E \rho(E) f(E) \rho(E+\hbar \omega)|\langle E+\hbar \omega|Q| E\rangle|^{2}
$$

and

$$
\frac{2}{\pi} \frac{\hbar \omega}{2}\left[\frac{1}{e^{\beta \hbar \omega}-1}\right] R(\omega)=\hbar \int_{0}^{\infty} d E \rho(E) f(E) \rho(E-\hbar \omega)|\langle E-\hbar \omega|Q| E\rangle|^{2}
$$


where $f(E)$ stands for a normalized Boltzmann factor with $f(E+\hbar \omega) / f(E)=$

$e^{-\beta \hbar \omega}$ and $\beta=1 / k T$. These formulas are remarkable: in the right-hand sides of these relations, we simply use the Fermi's golden rule for the first order perturbation. We recognize the left-hand side of (1) as representing the spontaneous and induced emissions of second-quantized Bosonic oscillators, and the left-hand side of (2) as the standard (induced ) absorption formula. Moreover the spectrum of the effective Bosonic oscillators is characterized by the dissipative coefficient ( resistance) $R(\omega)$. In other words, the presence of $R(\omega)$ necessarily implies the presence of effective oscillators characterized by $R(\omega)$, as was emphasized in Ref. 10. These expressions realize the basic idea of Feynman-Vernon[7] and Caldeira-Leggett [11] without actually introducing an infinite number of oscillators, and they naturally satisfiy the detailed balancing relation. It is shown that these formulas (1) and (2) lead to the conventional fluctuation-dissipation theorem of Callen and Welton.

Combining (1) and (2) with a dispersion relation, it is shown in the text that we can reproduce the tunneling formula in the presence of dissipation at finite temperature without referring to an explicit model Lagrangian.

We also present a generalization of the Callen-Welton formula for fermionic dissipation ( or fluctuation) in contrast to the conventional Bosonic dissipation such as in (1) and (2). These formulas for fermionic dissipation, though somewhat academic at this moment, may become relevant in the analyses of , for example, fermion emission from a black hole or supersymmetric properties at the early universe.

\section{Reformulation of fluctuation-dissipation theorem}

\subsection{Microscopic power dissipation}

We first start with a Hamiltonian

$$
H=H_{0}(Q)+V(Q, q) e^{i \omega t}+V(Q, q)^{\dagger} e^{-i \omega t}
$$

where $H_{0}(Q)$ is the unperturbed Hamiltonian for the dissipative medium, which has eigenstates

$$
H_{0}\left|E_{n}\right\rangle=E_{n}\left|E_{n}\right\rangle
$$


The variable $q$ appearing in $V(Q, q)$ describes the external dynamical freedom which perturbs the dissipative medium. The variable $q$ is treated as a classical variable for the moment.

If the dissipative medium is initially in the state $\left|E_{n}\right\rangle$, the lowest order transition rate (transition probability per unit time) is given by the Fermi's golden rule by treating the last two terms in (3) as small perturbation,

$$
\begin{aligned}
w=\frac{2 \pi}{\hbar}\left[\mid\left\langle E_{n}\right.\right. & \left.+\hbar \omega\left|V^{\dagger}\right| E_{n}\right\rangle\left.\right|^{2} \rho\left(E_{n}+\hbar \omega\right) \\
& \left.+\left|\left\langle E_{n}-\hbar \omega|V| E_{n}\right\rangle\right|^{2} \rho\left(E_{n}-\hbar \omega\right)\right] .
\end{aligned}
$$

Since the first term stands for the absorption of energy $\hbar \omega$ and the second term for the emission of energy $\hbar \omega$, the energy absorption rate by the dissipative medium is given by

$$
2 \pi \omega\left[\left|\left\langle E_{n}+\hbar \omega\left|V^{\dagger}\right| E_{n}\right\rangle\right|^{2} \rho\left(E_{n}+\hbar \omega\right)-\left|\left\langle E_{n}-\hbar \omega|V| E_{n}\right\rangle\right|^{2} \rho\left(E_{n}-\hbar \omega\right)\right] .
$$

If the system is initially in thermal equilibrium at temperature $T$, we must average over all initial states, weighting the state $\left|E_{n}\right\rangle$ by the (normalized) Boltzmann factor $f\left(E_{n}\right)$ which satisfies

$$
\frac{f\left(E_{n}+\hbar \omega\right)}{f\left(E_{n}\right)}=e^{-\beta \hbar \omega}, \quad \beta=\frac{1}{k T} .
$$

Then the energy dissipation per unit time is given by

$$
\begin{gathered}
P(\omega)=2 \pi \omega \int_{0}^{\infty} d E \rho(E) f(E)\left[\left|\left\langle E+\hbar \omega\left|V^{\dagger}\right| E\right\rangle\right|^{2} \rho(E+\hbar \omega)\right. \\
\left.-|\langle E-\hbar \omega|V| E\rangle|^{2} \rho(E-\hbar \omega)\right] .
\end{gathered}
$$

where we replaced the summation over $n$ by an integration over energy.

Now, we assume that $V$ can be written as $V=q Q / 2$, namely, the interaction part in (3) is written as

$$
H_{I}=\frac{1}{2}\left(q e^{i \omega t} Q+Q^{\dagger} \bar{q} e^{-i \omega t}\right)
$$

in the spirit of linear response approximation; $q$ is an infinitesimal complex number and $Q$ is a bosonic composite operator, respectively. Here we allow 
the operator $Q$ to be non-hermitian in general so that we can readily extend our formulation to fermionic dissipation later. Then, we obtain

$$
\begin{aligned}
P(\omega)=\frac{\pi \omega}{2} q \bar{q} \int_{0}^{\infty} & d E \rho(E) f(E)\left[\left|\left\langle E+\hbar \omega\left|Q^{\dagger}\right| E\right\rangle\right|^{2} \rho(E+\hbar \omega)\right. \\
& \left.-|\langle E-\hbar \omega|Q| E\rangle|^{2} \rho(E-\hbar \omega)\right] .
\end{aligned}
$$

\subsection{Macroscopic dissipative coefficient $R(\omega)$}

We next define the phenomenological macroscopic dissipative coefficient (resistance) $R(\omega)$ on the basis of the following reasoning [10]. We first define an infinitesimal ( complex ) coordinate in $H_{I}(9)$

$$
q(t)=q e^{i \omega t}
$$

The existence of the energy dissipation into the medium (10) induced by the external perturbation $q(t)$ suggests the presence of a dissipative force (reaction) acting on the variable $\operatorname{Req}(t)$, which oscillates with frequency $\omega$,

$$
F=-R(\omega) \operatorname{Re} \dot{q}(t)
$$

where $R(\omega)$ is a real function. Note that $H_{I}=Q R e q(t)$ in (9) for a hermitian operator $Q$; this shows that $R e q(t)$ is a natural classical counter part of the ( hermitian ) quantum variable $\hat{q}$ to describe the macroscopic quantum system $H_{0}(q)$.

The power dissipation per unit time generated by this phenomenological reactive force is given by

$$
\begin{aligned}
P(\omega) & =-\overline{\operatorname{ReFRe} \dot{q}(t)} \\
& =R(\omega) \overline{(\operatorname{Re} \dot{q}(t))^{2}} \\
& =\frac{1}{2} R(\omega) \overline{(\operatorname{Re} \dot{q}(t))^{2}+(\operatorname{Im} \dot{q}(t))^{2}} \\
& =\frac{\omega^{2}}{2} R(\omega) q \bar{q}
\end{aligned}
$$

where the overline indicates time averaging. 
Combining (10) and (13), we obtain the microscopic expression for the dissipative coefficient ( resistance ) $R(\omega)$

$$
\begin{aligned}
R(\omega)= & \frac{\pi}{\omega} \int_{0}^{\infty} d E \rho(E) f(E)\left[\left|\left\langle E+\hbar \omega\left|Q^{\dagger}\right| E\right\rangle\right|^{2} \rho(E+\hbar \omega)\right. \\
& \left.\quad-|\langle E-\hbar \omega|Q| E\rangle|^{2} \rho(E-\hbar \omega)\right] \\
= & \frac{\pi}{\omega}\left(1-e^{-\beta \hbar \omega}\right) \int_{0}^{\infty} d E \rho(E) f(E) \rho(E+\hbar \omega)\left|\left\langle E+\hbar \omega\left|Q^{\dagger}\right| E\right\rangle\right|^{2} .
\end{aligned}
$$

From this expression of $R(\omega)$, we find the basic relations,

$$
\begin{aligned}
\frac{2}{\pi} \frac{\hbar \omega}{2}\left[1+\frac{1}{e^{\beta \hbar \omega}-1}\right] R(\omega) & =\hbar \int_{0}^{\infty} d E \rho(E) f(E) \rho(E+\hbar \omega)\left|\left\langle E+\hbar \omega\left|Q^{\dagger}\right| E\right\rangle\right|^{2}, \\
\frac{2}{\pi} \frac{\hbar \omega}{2}\left[\frac{1}{e^{\beta \hbar \omega}-1}\right] R(\omega) & =\hbar \int_{0}^{\infty} d E \rho(E) f(E) \rho(E-\hbar \omega)|\langle E-\hbar \omega|Q| E\rangle|^{2} .
\end{aligned}
$$

Eq.(15) stands for the absorption of $\hbar \omega$ by $Q^{\dagger}$ and Eq.(16) stands for the emission of $\hbar \omega$ by $Q$.

These relations (15) and (16) stand for the proto-type of the fluctuationdissipation theorem of Callen and Welton. The fluctuation-dissipation theorem as it stands is a mathematical identity and contains no physical contents by itself. What is remarkable is that we obtain highly non-trivial relations in (15) and (16) if one assumes that the dissipative coefficient $R(\omega)$ little depends on the temperature in the region we are interested in. We can recognize the left-hand side of (15) as standing for the spontaneous and induced emissions of the second quantized bosonic oscillator with frequency $\omega$ into the dissipative medium, whereas the left-hand side of (16) is recognized as the (induced) absorption of these oscillators from the dissipative medium at temperature $T$. Moreover, the spectrum of these effective oscillators is precisely specified by the dissipative coefficient $R(\omega)$. In particular, there is no effective oscillators of $\omega$ for which $R(\omega)$ vanishes. These properties realize the basic physical idea of Feynman-Vernon [7] and Caldeira-Leggett [11], which simulates the dissipative medium by an infinite number of oscillators. In our approach this physical idea is realized by a simple application of the Fermi's 
golden rule combined with the temperature independence of the dissipative coefficient $R(\omega)$ without actually introducing oscillators. We emphasize that these oscillators are effective ones and there do not exist such real oscillators inside the dissipative medium in general. It is shown in next section that the quantum tunneling with dissipation at finite temperature is formulated on the basis of (15) and (16) and dispersion relations (i.e., unitarity and causality) without referrring to the explicit model Lagrangian of Caldeira and Leggett.

It is obvious from Eq.(3) that the change $\omega \rightarrow-\omega$ corresponds to the exchange $V \leftrightarrow V^{\dagger}$. By definition of $R(\omega)$ in (46), we then obtain the expression

$$
\begin{aligned}
R(-\omega)= & \frac{\pi}{\omega} \int_{0}^{\infty} d E \rho(E) f(E)\left[|\langle E+\hbar \omega|Q| E\rangle|^{2} \rho(E+\hbar \omega)\right. \\
& \left.\quad-\left|\left\langle E-\hbar \omega\left|Q^{\dagger}\right| E\right\rangle\right|^{2} \rho(E-\hbar \omega)\right] \\
= & \frac{\pi}{\omega}\left(1-e^{-\beta \hbar \omega}\right) \int_{0}^{\infty} d E \rho(E) f(E) \rho(E+\hbar \omega)|\langle E+\hbar \omega|Q| E\rangle|^{2}
\end{aligned}
$$

Clearly, $R(-\omega)=R(\omega)$ in the case of a hermitian operator, $Q=Q^{\dagger}$.

For $R(-\omega)$ with $\omega>0$, we obtain relations similar to (15) and (16),

$$
\begin{aligned}
\frac{2}{\pi} \frac{\hbar \omega}{2}\left[1+\frac{1}{e^{\beta \hbar \omega}-1}\right] R(-\omega) & =\hbar \int_{0}^{\infty} d E \rho(E) f(E) \rho(E+\hbar \omega)|\langle E+\hbar \omega|Q| E\rangle|^{2}, \\
\frac{2}{\pi} \frac{\hbar \omega}{2}\left[\frac{1}{e^{\beta \hbar \omega}-1}\right] R(-\omega) & =\hbar \int_{0}^{\infty} d E \rho(E) f(E) \rho(E-\hbar \omega)\left|\left\langle E-\hbar \omega\left|Q^{\dagger}\right| E\right\rangle\right|^{2} .
\end{aligned}
$$

Eq.(19) stands for the absorption of $\hbar \omega$ by $Q$ and Eq.(20) stands for the emission of $\hbar \omega$ by $Q^{\dagger}$ from the dissipative medium, respectively.

\subsection{Fluctuation-dissipation theorem}

Finally, we formulate the fluctuation-dissipation theorem of Callen and Welton. From Eqs.(15) and (20), we have after integration over $\omega$ ( and changing the order of integration over $E$ and $\omega$ )

$$
\left\langle Q Q^{\dagger}\right\rangle \equiv \int_{0}^{\infty}\left\langle E\left|Q Q^{\dagger}\right| E\right\rangle \rho(E) f(E) d E
$$




$$
\begin{aligned}
= & \int_{0}^{\infty} d E \rho(E) f(E)\left\{\int_{0}^{\infty}\left|\left\langle E+\hbar \omega\left|Q^{\dagger}\right| E\right\rangle\right|^{2} \rho(E+\hbar \omega) d(\hbar \omega)\right. \\
& \left.+\int_{0}^{\infty}\left|\left\langle E-\hbar \omega\left|Q^{\dagger}\right| E\right\rangle\right|^{2} \rho(E-\hbar \omega) d(\hbar \omega)\right\} \\
= & \frac{2}{\pi} \int_{0}^{\infty} d \omega \frac{\hbar \omega}{2}\left\{\left[1+\frac{1}{e^{\beta \hbar \omega}-1}\right] R(\omega)+\left[\frac{1}{e^{\beta \hbar \omega}-1}\right] R(-\omega)\right\}
\end{aligned}
$$

and similarly from Eqs.(16) and (19),

$$
\left\langle Q^{\dagger} Q\right\rangle=\frac{2}{\pi} \int_{0}^{\infty} d \omega \frac{\hbar \omega}{2}\left\{\left[\frac{1}{e^{\beta \hbar \omega}-1}\right] R(\omega)+\left[1+\frac{1}{e^{\beta \hbar \omega}-1}\right] R(-\omega)\right\}
$$

Furthermore,

$$
\frac{1}{2}\left\langle Q^{\dagger} Q+Q Q^{\dagger}\right\rangle=\frac{2}{\pi} \int_{0}^{\infty} d \omega E(\omega, T)\left[\frac{R(\omega)+R(-\omega)}{2}\right],
$$

where

$$
E(\omega, T)=\frac{\hbar \omega}{2}+\frac{\hbar \omega}{e^{\beta \hbar \omega}-1} .
$$

If $\mathrm{Q}$ is a hermitian operator, $Q=Q^{\dagger}$, we have $R(-\omega)=R(\omega)$, and Eqs.(21)(23) all reduce to the familiar form of the fluctuation-dissipation theorem of Callen and Welton[2],

$$
\left\langle Q^{2}\right\rangle=\frac{2}{\pi} \int_{0}^{\infty} d \omega E(\omega, T) R(\omega) .
$$

The fluctuation $\left\langle Q^{2}\right\rangle$ in the left-hand side is expressed in terms of the dissipative coefficient $R(\omega)$ with a universal kernel $E(\omega, T)$. The fluctuationdissipation theorem is regarded as a sum rule relating two quantities $\left\langle Q^{2}\right\rangle$ and $R(\omega)$, which are measured independently.

In Ref. [10], we used the final formula (25) and thus only the zero temperature case of quantum tunneling with dissipation was formulated in a model independent manner. The relations (15) and (16) apparently contain more information, and they allow us to formulate the tunneling with dissipation at finite temperature. 


\section{Quantum tunneling with dissipation at fi- nite temperature}

We start with the total Hamiltonian

$$
H=H_{0}(Q)+H_{0}(q)+H_{I}(q, Q)
$$

where $H_{0}(q)$ describes the unperturbed Hamiltonian of the quantum system we are interested in

$$
H_{0}(q)=\frac{1}{2 M} p^{2}+V(q)
$$

and $H_{I}(q, Q)$ stands for the interaction Hamiltonian in (10)

$$
H_{I}(q, Q)=q Q
$$

but now the variable $q$ is promoted to a hermitian quantum operator; the explicit time dependence disappears in the Schroedinger picture. We also choose a hermitian $Q, Q^{\dagger}=Q$, in conformity with the convention of the standard Caldeira-Leggett model [11]. In the context of macroscopic quantum tunneling (or to be more precise, quantum coherence), we choose $V(q)$ in (28) as standing for a symmetric double well potential. $H_{0}(Q)$ describes the dissipative medium as before, but we do not need an explicit form of $H_{0}(Q)$ and the dynamical properties of $H_{0}(Q)$ are indirectly specified by our fluctuation-dissipation theorem (15) and (16).

We now start with the eigenstates of $H_{0}(q)$

$$
H_{0}(q)|n\rangle=E_{n}|n\rangle
$$

and consider the transition probability for $n \rightarrow m+\hbar \omega$ by emitting energy $\hbar \omega$ to the dissipative medium, which is assumed to be in thermal equilibrium with temperature $T$. The transition probability for this process is given by the lowest order perturbation of $H_{I}(q, Q)$ as

$$
\begin{aligned}
& w(n \rightarrow m+\hbar \omega) \\
= & \frac{2 \pi}{\hbar}|\langle m|q| n\rangle|^{2} \int_{0}^{\hbar \Lambda}\left[\int_{0}^{\infty} \rho(E+\hbar \omega)|\langle E+\hbar \omega|Q| E\rangle|^{2} \rho(E) f(E) d E\right] \\
& \times \delta\left(E_{n}-E_{m}-\hbar \omega\right) d(\hbar \omega) \\
= & \frac{2 \pi}{\hbar}|\langle m|q| n\rangle|^{2} \frac{2}{\pi} \int_{0}^{\hbar \Lambda} \frac{\hbar \omega}{2}\left[1+\frac{1}{e^{\hbar \omega / k T}-1}\right] \frac{R(\omega)}{\hbar} \delta\left(E_{n}-E_{m}-\hbar \omega\right) d(\hbar \omega)
\end{aligned}
$$


where we used eq.(15) for a hermitian $Q$. We also introduced an explicit cut-off $\hbar \Lambda$ of effective frequency of the dissipative medium, which could be included in the definition of $R(\omega)$.

At finite temperature we also have an absorption probability

$$
\begin{aligned}
& w(n+\hbar \omega \rightarrow m) \\
= & \frac{2 \pi}{\hbar}|\langle m|q| n\rangle|^{2} \int_{0}^{\hbar \Lambda}\left[\int_{0}^{\infty} \rho(E-\hbar \omega)|\langle E-\hbar \omega|Q| E\rangle|^{2} \rho(E) f(E) d E\right] \\
& \times \delta\left(E_{n}-E_{m}+\hbar \omega\right) d(\hbar \omega) \\
= & \frac{2 \pi}{\hbar}|\langle m|q| n\rangle|^{2} \frac{2}{\pi} \int_{0}^{\hbar \Lambda} \frac{\hbar \omega}{2}\left[\frac{1}{e^{\hbar \omega / k T}-1}\right] \frac{R(\omega)}{\hbar} \delta\left(E_{n}-E_{m}+\hbar \omega\right) d(\hbar \omega)
\end{aligned}
$$

where we used (16). It is interesting that these formulas satisfy the detailed balancing relation

$$
e^{\hbar \omega / k T} w(n \rightarrow m+\hbar \omega)=w(m+\hbar \omega \rightarrow n)
$$

with $\hbar \omega=E_{n}-E_{m}$.

We next define the half-width of the state $|n\rangle$ for emission( when we prepare the state $|n\rangle$ at $t=0)$

$$
\frac{1}{2} \Gamma_{n}^{(+)}=\frac{1}{2} \hbar \sum_{m} w(n \rightarrow m+\hbar \omega)
$$

and the corresponding one for absorption

$$
\frac{1}{2} \Gamma_{n}^{(-)}=\frac{1}{2} \hbar \sum_{m} w(n+\hbar \omega \rightarrow m)
$$

It can be confirmed that eq. (33) gives $(1 / 2) \Gamma_{n}=(1 / 2) \hbar \eta / M$ at $T=0$ for a simple harmonic oscillator $H_{0}(q)=(1 / 2 M) p^{2}+\left(M \omega^{2} / 2\right) q^{2}$ and Ohmic dissipation $R(\omega)=\eta[10]$; this expression of $\Gamma_{n}$ is consistent with a damped oscillator $M \ddot{q}+\eta \dot{q}+M \omega^{2} q=0$, which in turn justifies the normalization of $H_{I}$ in (28).

The basic idea in our attempt to reproduce the results of the CaldeiraLeggett model without introducing an auxiliary infinite number of oscillators is to write dispersion relations, which relate the imaginary part of energy 
eigenvalue to the corresponding real part. The imaginary parts are evaluated by means of the fluctuation-dissipation theorem as in (33) and (34).

We thus write a generalization of the dispersion relation for the self-energy correction $\Sigma_{n}(E)$ to the energy eigenvalue $E_{n}$ as

$$
\begin{aligned}
\Sigma_{n}(E) & =\frac{1}{\pi} \int_{0}^{\infty} \frac{\operatorname{Im} \Sigma_{n}^{(+)}\left(E^{\prime}\right) d E^{\prime}}{E^{\prime}-E-i \epsilon} \\
& +\frac{1}{\pi} \int_{-\hbar \Lambda}^{\infty} \frac{\operatorname{Im} \Sigma_{n}^{(-)}\left(E^{\prime}\right) d E^{\prime}}{E^{\prime}-E-i \epsilon}
\end{aligned}
$$

where

$$
\begin{aligned}
\operatorname{Im} \Sigma_{n}^{(+)}(E) & \equiv \frac{1}{2} \Gamma_{n}^{(+)}(E) \\
& =\sum_{m}|\langle m|q| n\rangle|^{2} \int_{0}^{\hbar \Lambda} \hbar \omega\left[1+\frac{1}{e^{\hbar \omega / k T}-1}\right] \frac{R(\omega)}{\hbar} \delta\left(E-E_{m}-\hbar \omega\right) d(\hbar \omega)
\end{aligned}
$$

and

$$
\begin{aligned}
\operatorname{Im} \Sigma_{n}^{(-)}(E) & \equiv \frac{1}{2} \Gamma_{n}^{(-)}(E) \\
& =\sum_{m}|\langle m|q| n\rangle|^{2} \int_{0}^{\hbar \Lambda} \hbar \omega\left[\frac{1}{e^{\hbar \omega / k T}-1}\right] \frac{R(\omega)}{\hbar} \delta\left(E-E_{m}+\hbar \omega\right) d(\hbar \omega)
\end{aligned}
$$

Note that the lower bound of the integration range in the second term in eq.(35) starts at $-\hbar \Lambda$ due to the definition in (37). We thus obtain

$$
\begin{aligned}
\Sigma_{n}(E) & =\sum_{m}|\langle m|q| n\rangle|^{2} \frac{1}{\pi} \int_{0}^{\hbar \Lambda}\left\{\frac{\hbar \omega}{E_{m}+\hbar \omega-E-i \epsilon}\left[1+\frac{1}{e^{\hbar \omega / k T}-1}\right]\right. \\
& \left.+\frac{\hbar \omega}{E_{m}-\hbar \omega-E-i \epsilon}\left[\frac{1}{e^{\hbar \omega / k T}-1}\right]\right\} \frac{R(\omega)}{\hbar} d(\hbar \omega)
\end{aligned}
$$

\section{Ohmic dissipation}

For the Ohmic dissipation, $R(\omega) \equiv \eta=$ constant, in which we specialize from now on, we have the real part of the energy shift from (38) as

$$
\operatorname{Re} \Sigma_{n}\left(E_{n}\right)
$$




$$
\begin{aligned}
& =\frac{\eta}{\hbar \pi} \sum_{m}|\langle m|q| n\rangle|^{2} \hbar \Lambda \\
& -\frac{\eta}{\hbar \pi} \sum_{m}|\langle n|q| m\rangle|^{2}\left(E_{m}-E_{n}\right) \int_{0}^{\hbar \Lambda} P\left\{\frac{1}{\hbar \omega+E_{m}-E_{n}}\right. \\
& \left.+\left[\frac{1}{\hbar \omega+E_{m}-E_{n}}+\frac{1}{\hbar \omega-E_{m}+E_{n}}\right] \frac{1}{e^{\hbar \omega / k T}-1}\right\} d(\hbar \omega)
\end{aligned}
$$

where $P$ stands for the principal value prescription. After subtracting the first term proportional to $\hbar \Lambda$ as a renormalization of the potential [13 following the prescription of Caldeira and Leggett [11, the real part $\operatorname{Re} \Sigma_{n}\left(E_{n}\right)$ is rewritten as

$$
\begin{aligned}
& \operatorname{Re} \Sigma_{n}\left(E_{n}\right)=-\frac{\eta}{\hbar \pi} \sum_{m}|\langle n|q| m\rangle|^{2}\left(E_{m}-E_{n}\right) \\
& \times \int_{0}^{\hbar \Lambda} \frac{1}{2}\left\{\left[\frac{1}{\hbar \omega+E_{m}-E_{n}}+\frac{1}{\hbar \omega-E_{m}+E_{n}}\right]\left(1+\frac{2}{e^{\hbar \omega / k T}-1}\right)\right\} d(\hbar \omega) \\
& =-\frac{\eta}{\hbar \pi} \sum_{m}|\langle n|q| m\rangle|^{2}\left(E_{m}-E_{n}\right) \\
& \times \int_{0}^{\hbar \Lambda} \frac{1}{2} P\left[\frac{1}{\hbar \omega+E_{m}-E_{n}}+\frac{1}{\hbar \omega-E_{m}+E_{n}}\right] \operatorname{coth}\left(\frac{\beta \hbar \omega)}{2}\right) d(\hbar \omega)
\end{aligned}
$$

which agrees with the result of the field theoretical formulation of the Caldeira-Leggett model[13]. It should be noted that the vacuum fluctuation term ( spontaneous emission ) in (1) plays a central role in our application, unlike the conventional applications of the fluctuation- dissipation theorem where the vacuum fluctuation is usually subtracted [2].

For $\beta=1 / k T \rightarrow \infty$, one naturally recovers the zero temperature result 10. For the two-level approximation, which is valid for the lowest two levels in a deep double well potential, we have the result 13.

$$
\operatorname{Re} \Sigma_{2}\left(E_{2}\right)-\operatorname{Re} \Sigma_{1}\left(E_{1}\right) \simeq \epsilon \bar{\eta} \ln \left(e^{-2} \beta \hbar \Lambda\right)
$$

with the zeroth order energy difference $\epsilon \equiv E_{2}-E_{1}$ and $\bar{\eta}=\frac{2 \eta}{\pi \hbar}|\langle 0|q| 1\rangle|^{2}$ for the temperature $\epsilon \ll 1 / \beta \ll \hbar \Lambda$. One can confirm the absence of $\epsilon \ln \epsilon$ dependence by splitting the integration range in (40) into $[0, \hbar \Lambda]=[0, a]+$ $[a, \hbar \Lambda]$ with $\epsilon \ll a \ll 1 / \beta \ll \hbar \Lambda$. The energy splitting ( order parameter of quantum coherence) corrected by the dissipation is then given by

$$
\epsilon_{(1)}=\left(E_{2}-\operatorname{Re} \Sigma_{2}\left(E_{2}\right)\right)-\left(E_{1}-\operatorname{Re} \Sigma_{1}\left(E_{1}\right)\right)
$$




$$
\begin{aligned}
& \simeq \epsilon\left[1-\bar{\eta} \ln \left(e^{-2} \beta \hbar \Lambda\right)\right] \\
& \simeq \epsilon\left[\left(e^{-2}\right) \beta \hbar \Lambda\right]^{-\bar{\eta}}
\end{aligned}
$$

after the renormalization group improvement. This result, which suggests the suppression of quantum coherence $\epsilon_{(1)} \ll \epsilon$ for $\beta \hbar \Lambda \gg 1$, is in agreement with the dilute instanton analysis for the case of Ohmic dissipation 12]. Eq.(42), when compared with the result $\epsilon_{(1)}=\epsilon\left[\hbar \omega_{0} / \epsilon\right]^{-\bar{\eta}}$ or $\epsilon_{(1)}=\epsilon\left[\hbar \omega_{0} / \epsilon\right]^{-\bar{\eta} /(1-\bar{\eta})}$ at $T=0[12]$ [13, shows that the infrared cut-off, which was originally provided by $\epsilon$, is replaced by $1 / \beta$. We can thus analyze the quantum coherence without referring to an explicit model Lagrangian.

Although the mathematical basis of the dispersion relation at finite temperature is less solid compared with the one at zero temperature, our relation (35) is justisfied in the present linear response approximation in a limited temperature region since it coincides with the second order perturbation theory combined with (15) and (16)(or (30) and (31)); we note that the diagonal matrix element $\langle n|q| n\rangle=0$ for a specific double-well potential.

\section{Fluctuation-dissipation theorem for fermionic dissipation}

We now discuss a fermionic version of the fluctuation-dissipation theorem of Callen and Welton starting with eq.(9). The operators $Q$ and $Q^{\dagger}$ are now taken to be fermionic operators and $q$ and $\bar{q}$ are Grassmann numbers which satisfy

$$
q \bar{q}=-\bar{q} q, \quad q^{2}=0, \quad \bar{q}^{2}=0
$$

and

$$
Q q=-q Q, \quad Q \bar{q}=-\bar{q} Q, \quad Q^{2}=0, \quad\left(Q^{\dagger}\right)^{2}=0
$$

Because of $Q^{2}=0$, the real fermionic case is trivial, and we consider a complex $Q$ which satisfy $Q Q^{\dagger} \neq 0$. In a relativistic notation of 4-dimensional space-time, our $Q$ is regarded as one of the components of (composite) twocomponent complex spinor $Q_{\alpha}, \alpha=1,2$. (Instead, one may also consider a set

of real fermionic operators, $Q_{1}$ and $Q_{2}$ with $Q_{1}^{\dagger}=Q_{1}, Q_{2}^{\dagger}=Q_{2}$ corresponding to a Majorana spinor, which satisfy $\left.Q_{1} Q_{2} \neq 0\right)$. 
Eq.(10) is now replaced by

$$
\begin{gathered}
P(\omega)=\frac{\pi \omega}{2} q \bar{q} \int_{0}^{\infty} d E \rho(E) f(E)\left[\left|\left\langle E+\hbar \omega\left|Q^{\dagger}\right| E\right\rangle\right|^{2} \rho(E+\hbar \omega)\right. \\
\left.+|\langle E-\hbar \omega|Q| E\rangle|^{2} \rho(E-\hbar \omega)\right] .
\end{gathered}
$$

Note the relative sign of two terms in (45), which arises from the Grassmann nature of $q$ and $\bar{q}$. We adopt the definition of the dissipative coefficient $P(\omega)=\frac{\omega^{2}}{2} R_{f}(\omega) q \bar{q}$ as in eq.(13). We thus obtain

$$
\begin{aligned}
R_{f}(\omega)= & \frac{\pi}{\omega} \int_{0}^{\infty} d E \rho(E) f(E)\left[\left|\left\langle E+\hbar \omega\left|Q^{\dagger}\right| E\right\rangle\right|^{2} \rho(E+\hbar \omega)\right. \\
& \left.\quad+|\langle E-\hbar \omega|Q| E\rangle|^{2} \rho(E-\hbar \omega)\right] \\
= & \frac{\pi}{\omega}\left(1+e^{-\beta \hbar \omega}\right) \int_{0}^{\infty} d E \rho(E) f(E) \rho(E+\hbar \omega)\left|\left\langle E+\hbar \omega\left|Q^{\dagger}\right| E\right\rangle\right|^{2}
\end{aligned}
$$

From this expression of $R_{f}(\omega)$, we find the basic relations

$$
\begin{aligned}
\frac{2}{\pi} \frac{\hbar \omega}{2}\left[1-\frac{1}{e^{\beta \hbar \omega}+1}\right] R_{f}(\omega) & =\hbar \int_{0}^{\infty} d E \rho(E) f(E) \rho(E+\hbar \omega)\left|\left\langle E+\hbar \omega\left|Q^{\dagger}\right| E\right\rangle\right|^{2}, \\
\frac{2}{\pi} \frac{\hbar \omega}{2}\left[\frac{1}{e^{\beta \hbar \omega}+1}\right] R_{f}(\omega) & =\hbar \int_{0}^{\infty} d E \rho(E) f(E) \rho(E-\hbar \omega)|\langle E-\hbar \omega|Q| E\rangle|^{2} .
\end{aligned}
$$

The left-hand side of (47) is regarded as the spontaneous and induced emissions of (effective) fermionic oscillators, and the ralative minus sign accounts for the Fermi statistics. The left-hand side of (48) is regarded as the (induced) absorption of the fermionic oscillators.

Eq.(17) is now replaced by

$$
\bar{R}_{f}(-\omega)=\frac{\pi}{\omega}\left(1+e^{-\beta \hbar \omega}\right) \int_{0}^{\infty} d E \rho(E) f(E) \rho(E+\hbar \omega)|\langle E+\hbar \omega|Q| E\rangle|^{2} .
$$

where we defined $P(-\omega)=\frac{\omega^{2}}{2} \bar{R}_{f}(\omega) \bar{q} q$ by changing the order of $q$ and $\bar{q}$. For $\bar{R}_{f}(-\omega)$ with $\omega>0$, we thus obtain the relations

$$
\frac{2}{\pi} \frac{\hbar \omega}{2}\left[1-\frac{1}{e^{\beta \hbar \omega}+1}\right] \bar{R}_{f}(-\omega)=\hbar \int_{0}^{\infty} d E \rho(E) f(E) \rho(E+\hbar \omega)|\langle E+\hbar \omega|Q| E\rangle|^{2}
$$




$$
\frac{2}{\pi} \frac{\hbar \omega}{2}\left[\frac{1}{e^{\beta \hbar \omega}+1}\right] \bar{R}_{f}(-\omega)=\hbar \int_{0}^{\infty} d E \rho(E) f(E) \rho(E-\hbar \omega)\left|\left\langle E-\hbar \omega\left|Q^{\dagger}\right| E\right\rangle\right|^{2} .
$$

Combining these relations we finally obtain the fluctuation-dissipation theorem for fermionic dissipation ( or fluctuation) as

$$
\begin{aligned}
\left\langle Q Q^{\dagger}\right\rangle \equiv & \int_{0}^{\infty}\left\langle E\left|Q Q^{\dagger}\right| E\right\rangle \rho(E) f(E) d E \\
= & \int_{0}^{\infty} d E \rho(E) f(E)\left\{\int_{0}^{\infty}\left|\left\langle E+\hbar \omega\left|Q^{\dagger}\right| E\right\rangle\right|^{2} \rho(E+\hbar \omega) d(\hbar \omega)\right. \\
& \left.+\int_{0}^{\infty}\left|\left\langle E-\hbar \omega\left|Q^{\dagger}\right| E\right\rangle\right|^{2} \rho(E-\hbar \omega) d(\hbar \omega)\right\} \\
= & \frac{2}{\pi} \int_{0}^{\infty} d \omega \frac{\hbar \omega}{2}\left\{\left[1-\frac{1}{e^{\beta \hbar \omega}+1}\right] R_{f}(\omega)+\left[\frac{1}{e^{\beta \hbar \omega}+1}\right] \bar{R}_{f}(-\omega)\right\}
\end{aligned}
$$

and similarly

$$
\left\langle Q^{\dagger} Q\right\rangle=\frac{2}{\pi} \int_{0}^{\infty} d \omega \frac{\hbar \omega}{2}\left\{\left[\frac{1}{e^{\beta \hbar \omega}+1}\right] R_{f}(\omega)+\left[1-\frac{1}{e^{\beta \hbar \omega}+1}\right] \bar{R}_{f}(-\omega)\right\}
$$

Furthermore,

$$
\frac{1}{2}\left\langle Q^{\dagger} Q-Q Q^{\dagger}\right\rangle=\frac{2}{\pi} \int_{0}^{\infty} d \omega E_{f}(\omega, T)\left[\frac{R_{f}(\omega)-\bar{R}_{f}(-\omega)}{2}\right],
$$

where

$$
E_{f}(\omega, T)=-\frac{\hbar \omega}{2}+\frac{\hbar \omega}{e^{\beta \hbar \omega}+1} .
$$

Our sign convention of the dissipative coefficients $R_{f}(\omega)$ and $\bar{R}_{f}(-\omega)$ is chosen so that $R_{f}(\omega) \geq 0$ and $\bar{R}_{f}(-\omega) \geq 0$.

In practice, the composite operator $Q$ may carry a well-defined fermion number and the fermion number may be conserved. Moreover the production of an anti-particle ( or hole state ) may be suppressed; in such a case, one may set $\bar{R}_{f}(-\omega)=0$ in our formulas. The physical content of the fluctuationdissipation theorem for fermionic dissipation, as is formulated here, is that 
the thermal average of the (composite) operator $Q Q^{\dagger}$ or $Q^{\dagger} Q$, which characterizes the fluctuation, is represented in terms of effective fermionic excitations with their spectrum being specified by $R_{f}(\omega)$; the parameter $R_{f}(\omega)$ in turn characterizes the energy dissipation into the dissipative medium. In this context, the presence of energy dissipation with $R_{f}(\omega)$ necessarily leads to the presence of effective (or collective) fermionic excitations; this property is analogous to the Nambu-Goldstone theorem for spontaneous symmetry breakdown, which asserts an inevitable appearance of massless excitations when continuous symmetry is spontaneously broken, as was emphasized in Ref. 10. The difference is that these collective excitations in the present context are effective ones.

We finally comment on a technical complication in deriving (46). The calculation (46) may appear straightforward and identical to (14). However, a closer examination reveals that an additional assumption is in fact involved: If $|E\rangle$ is a bosonic state (with even "G parity" 15] ), i.e.,

$$
q|E\rangle=|E\rangle q
$$

then $Q|E\rangle$ is a fermionic state (odd G parity), i.e.,

$$
q(Q|E\rangle)=-(Q|E\rangle) q
$$

and vice versa. Since $\langle E \pm \hbar \omega|Q| E\rangle$ is not a Grassmann variable but rather an ordinary number, $|E \pm \hbar \omega\rangle$ should have different G parity from $|E\rangle$. That is, the interaction $q e^{i \omega t} Q+Q^{\dagger} \bar{q} e^{-i \omega t}$ would induce not only the energy shift $E \rightarrow$ $E \pm \hbar \omega$ but also the change of $\mathrm{G}$ parity. This fact prevents one from obtaining Eq.(46) in a naive way, because the state $|E\rangle$ obtained from $|E-\hbar \omega\rangle$ by a shift in $E, E \rightarrow E+\hbar \omega$, has a different G parity from the original $|E\rangle$. In obtaining (46) we assume that the initial state $|E\rangle$ has even $G$ parity with probability $1 / 2$ and odd $G$ parity with probability $1 / 2$. This assumption is consistent with the notion of dissipation, which implies that the "radiation" carries away or injects a small amount of energy specified by $\hbar \omega$ at a time. We can thus obtain Eq.(46) by averaging over G parity also.

\section{Conclusion}

The main purpose of the present paper is to point out the remarkable relations (1) and (2), which arise from a simple application of the Fermi's golden 
rule and the weak temperature dependence of the dissipative coefficient, and their applications to quantum tunneling with dissipation. In the conventional treatment of the fluctuation-dissipation theorem in (25), the vacuum fluctuation is often subtracted away by simply saying that it is not observable [14]. In contrast, the term corresponding to the vacuum fluctuation plays a central role in our application, since it describes the spontaneous emission of effective excitations into the dissipative medium; it is thus the only effect remaining at the vanishing temperature.

Our dispersion relation for the self-energy (35), which is essentially equivalent to the second order perturbation theory in linear response approximation, then gives rise to a change in the real part of the tunneling energy splitting ( which is the order parameter of quantum coherence). Our formulas naturally give rise to the dilute instanton results for the order parameter both at finite and vanishing temperature [12]. In this sense our attempt to reproduce the physical results of the Caldeira-Leggett model without referring to an explicit model Lagrangian has been successful at least in the analyses of quantum coherence, both at $T=0 \llbracket 10$ and $T \neq 0$.

Of course, the formulation of Caldeira and Leggett [11 is flexible enough and it is applicable to many other physical contexts. Nevertheless, for those who wonder if one can analyze some physical processes without an infinite number of oscillators, our reformulation of the quantum tunneling with dissipation on the basis of fluctuation-dissipation theorem and dispersion relations may give an answer by showing such possibility as well as limitations.

Another purpose of the present paper is to present a generalization of the fluctuation-dissipation theorem of Callen-Welton to the case of fermionic dissipation (or fluctuation). Since fermions are basically quantum mechanical, the notion of fermionic dissipation is characteristically quantum mechanical. We emphasize that a mere excitation of a fermion from one state to another does not imply fermionic fluctuation in the present context; the force fields or currents involved should be fermionic, though the fermionic excitation may be an effective one or a quasi-particle. In full quantum theory, both of the bosonic and fermionic modes can be equally added to or removed from the system we are interested in, and a fully quantum mechanical fluctuationdissipation theorem should be able to handle the fermionic fluctuation and, consequently, fermionic dissipation.

In the context of condensed matter physics, we usually measure bosonic quantities such as voltage or electric current even if the elementary process 
involves the transfer of fermions. In such a case, the conventional bosonic fluctuation-dissipation theorem is applicable. It is our hope that a properly defined treatment of elementary transfer processes may lead to an application of the notion of fermionic fluctuation or dissipation in the future.

As for the physical phenomena where the fermionic fluctuation-dissipation theorem may have some relevance, we note the fermion emission from a black hole [16] and the fermion production in an accelerated frame [17. The notion of quantum noise plays a fundamental role in the analyses of these processes, and thus the fermionic fluctuation-dissipation theorem may provide a convenient framework to describe some general features of these interesting quantum processes. Another area of physics where fermionic fluctuation may play a role is the theory of supersymmetry ( or Boson-Fermion symmetry) [18]. The basic current of supersymmetry is fermionic, and thus the thermal average of a product of such currents ( or related gravitino fields ) inevitably leads to a notion of fermionic fluctuation. Though we do not know a specific application of the fermionic fluctuation-dissipation theorem at this moment, our formulation may turn out to be useful in the future analyses of supersymmetry in a multi-particle thermal setting, where supersymmetry is known to be inevitably broken by thermal effects.

\section{References}

[1] L. Onsager, Phys. Rev. 37, 405(1931); 38, 2265(1931).

[2] H. B. Callen and T. A. Welton, Phys. Rev. 83, 34(1951).

[3] H. Takahasi, J. Phys. Soc. Jpn, 7, 439(1952).

[4] R. Kubo, J. Phys. Soc. Jpn, 12, 570(1957).

[5] H. Nakano, Int. J. Mod. Phys. B7, 2397(1993), and references therein.

[6] P. C. Martin and J. Schwinger, Phys. Rev. 115, 1342 (1959).

[7] R. P. Feynman and F. L. Vernon, Ann. of Phys. (N.Y.), 24, 118(1963).

[8] A. Einstein, Ann. Physik, 17, 549(1905).

[9] H. Nyquist, Phys. Rev. 32, 110(1928). 
[10] K. Fujikawa, Phys. Rev. E57 (1998)5023. hep-th/9802025.

[11] A. O. Caldeira and A. J. Leggett, Ann. of Phys., 149, 374(1983).

[12] A. O. Caldeira and A. J. Leggett, Phys. Rev. Lett. 46, 211 (1981); A. J. Bray and M. A. Moore, Phys. Rev. Lett. 49, 1545 (1982); S. Chakravarty, Phys. Rev. Lett. 49, 681 (1982); G. Schoen and A. D. Zaikin, Phys. Rep. 198, 237 (1990) and references therein.

[13] K. Fujikawa, S. Iso, M. Sasaki, and H. Suzuki, Phys. Rev. Lett. 68, 1093 (1992); Phys. Rev. B46, 10295 (1992).

[14] We thank S. Takagi for a discussion on the customary treatment of the vacuum fluctuation term appearing in the fluctuation-dissipation theorem.

[15] This usage of "G parity" is analogous to the GSO projection in the first quantization of superstring theory. See F. Gliozzi, J. Scherk, and D. Olive, Nucl. Phys. B122, 253(1977).

[16] R. M. Wald, General Relativity, (University of Chicago Press, Chicago, 1984), and references therein.

[17] S. Takagi, Prog. Theor. Phys. Suppl. 88, 1(1986), and references therein.

[18] J. Wess and J. Bagger, Supersymmetry and Supergravity (Princeton Univ. Press, 1983). 